\title{
Guest editorial: hybrid systems, part II
}

\author{
Yorai Wardi • Magnus Egerstedt
}

Published online: 14 January 2012

(C) Springer Science+Business Media, LLC 2012

It is a great pleasure for us to introduce this second volume on Hybrid Systems to Discrete Event Dynamic Systems: Theory and Applications. Hybrid dynamical systems are underscored by bi-layer dynamics, with time-driven dynamics at the lower layer and discrete-event dynamics at the upper layer. Such systems arise as abstract models in various application domains including switching circuits, mobile robotics, sensor networks, automotive powertrains, and more recently in cyber physical systems. They can be viewed as extensions of discrete-event dynamic systems that capture not only the event-driven dynamics but the time-driven dynamics as well, and the interactions between the two kinds of dynamics has provided a rich source of exciting research problems in design, control, and optimization.

The first part of this special issue contains five papers on a range of topics, including a tutorial on fluid Petri networks, optimal flow control in stochastic networks, stabilization of automata, control and optimization of a class of hybrid systems, and optimal scheduling in fluid queues (JDEDS, Vol. 21, No. 4, December 2011). The present, second part contains papers on hybrid traffic control, optimal control, abstractions of hybrid systems for complexity reduction in control, and a framework for compiling high-level control specifications into simple instructions.

The paper A Control Scheme for Freeway Traffic Systems Based on Hybrid Automata, by S. Sacone and S. Siri, devises a hybrid control law for freeway-traffic regulation. The control scheme has two parts: traffic lights at the on-ramps, and adaptive speed limits, displayed via message signs on the freeway. The control law is bi-layer with model-predictive control at the lower layer and a discrete-event

Y. Wardi $(\bowtie) \cdot$ M. Egerstedt

School of Electrical and Computer Engineering,

Georgia Institute of Technology, Atlanta, GA 30332, USA

e-mail: ywardi@ece.gatech.edu

M. Egerstedt

e-mail:magnus@ece.gatech.edu 
supervisory control at the upper layer. The resulting overall control scheme is described and analyzed in the setting of hybrid automata.

The paper Pseudo-Continuous Multi-Dimensional Multi-Mode Systems, by E.I. Verriest, concerns hybrid systems whose modes' state spaces have different dimensions. It focuses on the transitions between the modes, and especially on the lack of continuity of the state trajectories resulting from the different state spaces. It defines a weaker notion than continuity, called pseudo continuity, which is natural for characterizing regularity of the state trajectories at mode-transition instances. The paper discusses several control issues in the setting of such systems, and especially optimal control, where it characterizes optimality for a large class of systems. It illustrates this problem via an example of a spring-assisted high jump.

The paper A Proximal Point Based Approach to Optimal Control of Affine Switched Systems, by V. Azhmyakov, M. Basin, and J. Raisch, concerns optimal control problems on switched-mode hybrid systems. The dynamical systems associated with the various modes are described by nonlinear differential equations that are affine in the input signal. For this class of problems, the paper establishes continuity properties as well as their adequate approximation via convex optimization problems defined on Hilbert spaces. Finally, the paper proposes a numerical approach for solving the latter kind of problems.

The paper Finite Abstractions for Hybrid Systems with Stable Continuous Dynamics, by H.G. Tanner, J. Fu, C. Rawal, J.L. Piovesan, and C.T. Abdallah, considers the problem of model abstraction in hybrid systems and its application to control. The design of feedback control for hybrid systems can be highly complex, especially in the absence of observability. This paper investigates discrete, finite-transition abstractions of such systems, namely relatively-simple discrete models having the same properties as the hybrid systems. The paper focuses on a class of hybrid automata having limit sets, and it indicates how their abstractions can be used in the design of locally-stable control laws.

The paper Hybrid Systems Tools for Compiling Controllers for Cyber-Physical Systems, by P. Martin and M. Egerstedt, considers the gap between high-level specifications and particular implementations of control laws in cyber-physical systems. In capturing interactions between computing and communications systems on one hand, and their physical environments on the other hand, models of cyberphysical systems can have an enormous complexity. Furthermore, their control tasks often are described in terms of high-level attributes but their implementations require specific detailed instructions. This specification-to-execution gap is addressed in the present paper by a novel technique for compiling high-level control programs. The technique is described in the framework of hybrid systems, and it is suitable to control applications in a large class of systems.

The guest editors wish to thank the authors for their contributions, the reviewers for providing invaluable assessment and advice, and the editorial office for its support.

Yorai Wardi and Magnus Egerstedt 